\title{
Far-infrared spectroscopy of the field-induced spin-density-wave gap in (TMTSF) ${ }_{2} \mathrm{ClO}_{4}$
}

\author{
T. J. B. M. Janssen
}

High Field Magnet Laboratory, University of Nijmegen, Toernooiveld, NL-6525, ED Nijmegen, The Netherlands

A. S. Perel

Department of Physics, Boston University, Boston, Massachusetts 02215

and F. B. National Magnet Laboratory, Massachusetts Institute of Technology, 170 Albany Street, Cambridge, Massachusetts 02139

A. M. Gerrits

High Field Magnet Laboratory, University of Nijmegen, Toernooiveld, NL-6525, ED Nijmegen, The Netherlands

\author{
W. Kang \\ Department of Physics, Princeton University, Princeton, New Jersey 08544
}

J. S. Brooks

Department of Physics, Boston University, Boston, Massachusetts 02215

and F. B. National Magnet Laboratory, Massachusetts Institute of Technology, 170 Albany Street, Cambridge, Massachusetts 02139

\author{
A. Wittlin, J. A. A. J. Perenboom, and P. J. M. van Bentum* \\ High Field Magnet Laboratory, University of Nijmegen, Toernooiveld, NL-6525, ED Nijmegen, The Netherlands \\ (Received 1 May 1992)
}

\begin{abstract}
We report spectroscopic details of the excitation spectrum of the magnetic-field-induced spin-densitywave phases in the organic conductor (TMTSF) ${ }_{2} \mathrm{ClO}_{4}$ where TMTSF represents tetramethyltetraselenafulvalene. In contrast to theoretical predictions, the value of the energy gap is found to be constant in the different subphases, whereas the carrier density in the spin-density-wave state increases discontinuously upon each successive phase transition. In addition, we find evidence for a collective mode excitation in the microwave region, and a strong interaction of low-energy phonons with the spindensity-wave formation.
\end{abstract}

The charge-transfer salts of the Bechgaard family have a unique set of properties dependent on temperature, magnetic field, and pressure. ${ }^{1}$ The discovery of superconductivity in these materials marked the onset of extensive research, devoted to the understanding of the physical properties of these quasi-one-dimensional organic conductors. One consequence of the nearly sheetlike Fermi surface is the possibility of a nesting instability, leading to a spin (or charge) density wave. A special property of materials such as (TMTSF) ${ }_{2} \mathrm{ClO}_{4}$ is that a spin-density-wave state can be induced by applying a magnetic field. As a function of the applied field, the material undergoes a series of phase transitions with nearly quantized steps in the Hall voltage and related oscillations in the magnetoresistance, heat capacity, and magnetization. $^{2-5}$ Of paramount interest in these phase transitions are the induced changes in the electronic excitation spectrum. As in other strongly correlated condensates, such as charge-density-wave (CDW) systems and superconductors, the phase transition to spin-density-wave (SDW) state results also in the creation of a gap in the excitation spectrum. In the last decade a number of theories have been proposed to explain the anomalous behavior of this special class of charge-transfer salts. The magnetic-field-induced spin-density-wave state (FISDW) was first theoretically explained by Gor'kov and Lebed'. 6 Later $^{7,8}$ it was shown that the metallic phase was unstable for a quantized set of nesting vectors leading to a hierarchy of subphases. The understanding of the quantized Hall-like behavior was less straightforward because the band structure does not indicate any closed orbits on the Fermi surface and therefore a conventional Landaulevel quantization is not possible. The general understanding developed that the single-particle excitation spectrum in the FISDW phase consists of a series of Landau-like bands separated by more or less equidistant gaps. 9,10 The main SDW gap is predicted to occur at the Fermi level and, assuming that the carrier density is constant, one expects that this gap roughly follows the variations in the transition line $T_{c}(B) .^{11}$

Quinlivan et al. ${ }^{12}$ investigated the microwave and submillimeter properties of (TMTSF) ${ }_{2} \mathrm{PF}_{6}$, a related material, which has a SDW phase at zero magnetic field and ambient pressure.

Recent measurements by Perel et al. ${ }^{13}$ on (TMTSF) ${ }_{2} \mathrm{ClO}_{4}$ indicated the opening of a FISDW gap in the far-infrared (FIR) range. In this letter we present a detailed FIR and microwave study between 1.5 and 45 
$\mathrm{cm}^{-1}$ in fields up to $20 \mathrm{~T}$, covering both the metallic and FISDW states at a temperature of $400 \mathrm{mK}$. We report the first spectroscopic observation of the evolution of the excitation spectrum in the various spin-density subphases. The main result of this work is that a gap opens up that is independent of magnetic field or subphase index. We interpret this as an evidence that the gap is nonuniform in $k$ space and the gapped area on the Fermi surface appears to increase discontinuously upon each phase transition to a lower-index spin-density state. We will discuss additional observations related to a collective mode excitation in the microwave region and a coupling of low-energy phonons to the spin-density modulation.

The samples used in this work were prepared in a standard electrochemical way, yielding very pure crystals with a typical size of $0.5 \times 1 \times 7 \mathrm{~mm}^{3}$. In order to enhance the optical signal from these small crystals, we arranged a dozen crystals in a gridlike structure, so that the plane of the grid coincides with the $a b$ plane of the crystals. The samples were then placed in a ${ }^{3} \mathrm{He}$-cooled sample holder, mounted in the bore of either a $10-\mathrm{T}$ superconducting magnet or of a 20-T Bitter magnet. The magnetic-field direction was chosen along the $c$ axis, perpendicular to the grid. For this work we used different FIR setups; an optically pumped molecular FIR laser, providing approximately 20 lines in the submillimeter range $\left(10-30 \mathrm{~cm}^{-1}\right)$, several microwave klystrons in the frequency range between 30 and $70 \mathrm{GHz}\left(1-2.5 \mathrm{~cm}^{-1}\right)$, a set of microwave-pumped diode frequency multipliers, yielding a nearly continuous output between 2 and 15 $\mathrm{cm}^{-1}$ and a Bruker IFS 113 Fourier-transform IR spectrometer. All sources could be connected via lightpipes to the insert and were interchangeable without affecting the sample conditions. The combination of these different sources allows a quasicontinuous spectroscopy from the infrared range down to approximately $1.0 \mathrm{~cm}^{-1}$.

The FIR light intensity was measured with $\mathrm{Si}$ bolometers cooled to $1.2 \mathrm{~K}$. The sample was cooled at a rate of $30 \mathrm{mK} / \mathrm{min}$ through the anion-ordering transition at 24 $\mathrm{K}$ and was kept at $400 \mathrm{mK}$ during the experiment. Both reflection and transmission of the sample grid could be measured simultaneously, a technique first exploited by $\mathrm{Ng}$ et al. on these materials. ${ }^{14}$ Because of the good metallic conductivity along the $a$ axis, the reflectivity is close to unity and field-dependent changes in the extreme FIR are difficult to detect. The basic reason for using a grid arrangement is that transmission is restricted to a polarization along the less conducting $b$ axis and multiple reflections will enhance the effect of changes in the conductivity. Therefore the signal measured by the transmission bolometer basically corresponds to the sample reflectivity. A disadvantage of this configuration is that it is difficult to calibrate the absolute value of the sample absorption and also to perform a standard Kramers Kronig analysis. The linear polarization of the transmitted light in the spectral region of interest (below $30 \mathrm{~cm}^{-1}$ ) was confirmed in a separate experiment for temperatures down to $4 \mathrm{~K}$. A second sample was placed outside of the optical path but in good thermal contact with the holder. The magnetoresistance of this reference sample was used to monitor the formation of FISDW states.

Figure 1(a) shows the normalized transmission through the sample grid versus magnetic field for several monochromatic FIR energies. Here the transmission $(T)$ taken in the field is divided by the transmission in zero field. Large changes are found in $T(B) / T(0)$ at the various phase transitions. At this low temperature the transmission changes steplike with nearly-field-independent plateaus in between. With the help of the magnetoresistance data [Fig. 1(b)] taken on the reference sample these steps can be identified to correspond with transitions between different FISDW subphases. Above $7 \mathrm{~T}$ the spectra exhibited a large hysteresis depending on the magnetic-field history. Because of this effect only traces recorded with increasing field are shown. Some of the microwaveenergy $T(B) / T(0)$ traces are shown Fig. 2(b).

The most interesting aspect of these traces at constant radiation energy is that the changes near the phase transitions are quasisteplike, reminiscent of the steps in the Hall voltage. At low frequencies the sample reflectivity, proportional to $T(B) / T(0)$ generally decreases with field. It would indicate a smaller conductivity, in agreement with the opening of a (partial) gap in the excitation spectrum. A remarkable exception is the behavior in a narrow range around $2.4 \mathrm{~cm}^{-1}$, where $T(B) / T(0)$ sharp-
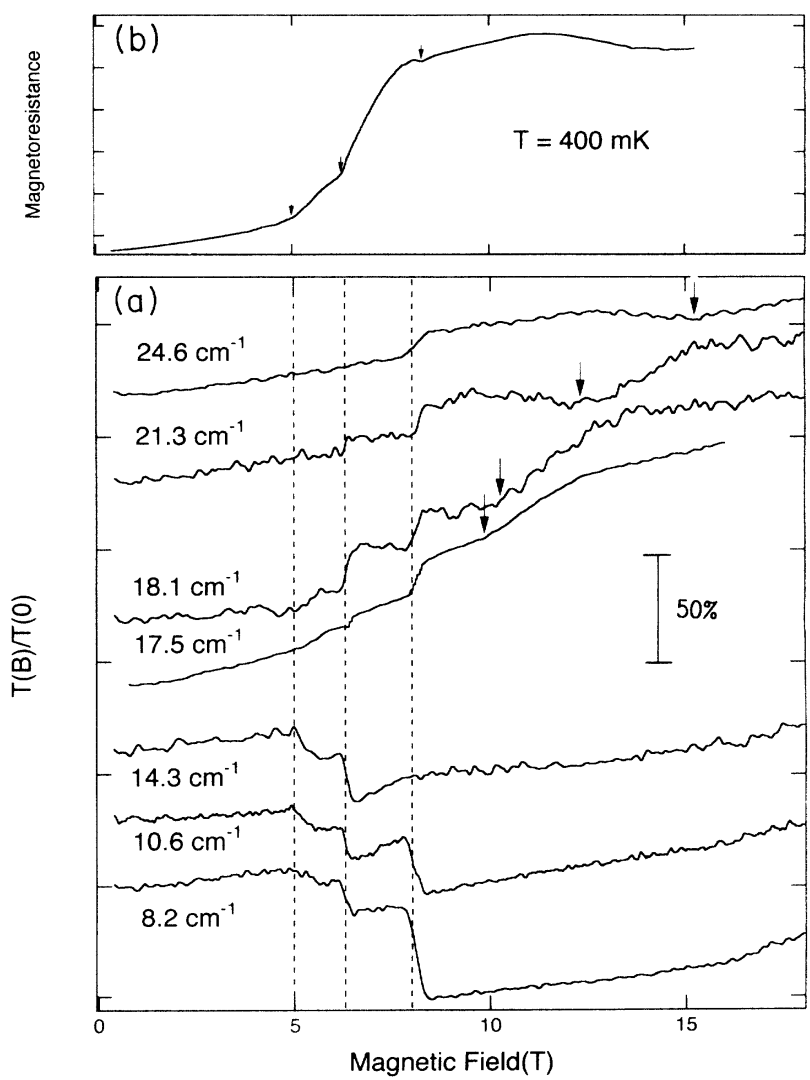

FIG. 1. (a) Transmission (normalized to transmission at $B=0)$ vs magnetic field for different energies both below and above the SDW gap. The arrows indicate the cyclotron resonance. Dashed lines correspond to fields where magnetoresistance steps were observed, as shown in (b). (b) Magnetoresistance of the reference sample. The arrows indicate the transitions between the FISDW subphases. 
ly increases. At frequencies above $14-\mathrm{cm}^{-1} T(B) / T(0)$ increases at the phase transitions, as one would expect when the single-particle density of states piles up above the gap edge. Normalized FIR spectra are shown in Fig. 2(a), showing the complete energy dependence. The spectra in magnetic field are normalized to the zero-field transmission spectrum. The data from the monochromatic energies are shown as points. For fields between the phase transitions the spectra were virtually identical, so we have limited the data to the three spectra that are shown in this figure. For historical reasons we labeled the FISDW phases with index $N=2,1,0$ corresponding to fields of $6,7.5$, and $8 \mathrm{~T}$. More spectra at fields up to $10 \mathrm{~T}$ have been taken, but these did not substantially differ from the 8-T spectra. From Fig. 2(a) several features can be identified: (a) a large decrease is found for $T(B) / T(0)$, below approximately $14 \mathrm{~cm}^{-1}$ in all FISDW phases, indicating the opening of a (partia!) gap. Note that this gap energy is the same in all subphases. (b) Sharp spectral features at 17 and $32 \mathrm{~cm}^{-1}$ appear suddenly with the formation of each SDW subphase. A smaller structure appears near $23 \mathrm{~cm}^{-1}$. (c) Finally, a resonance peak at $2.4 \mathrm{~cm}^{-1}$ is found. All features become more pronounced at higher fields or equivalently for lower FISDW index; however, none of them tend to shift in energy with increasing magnetic fields.
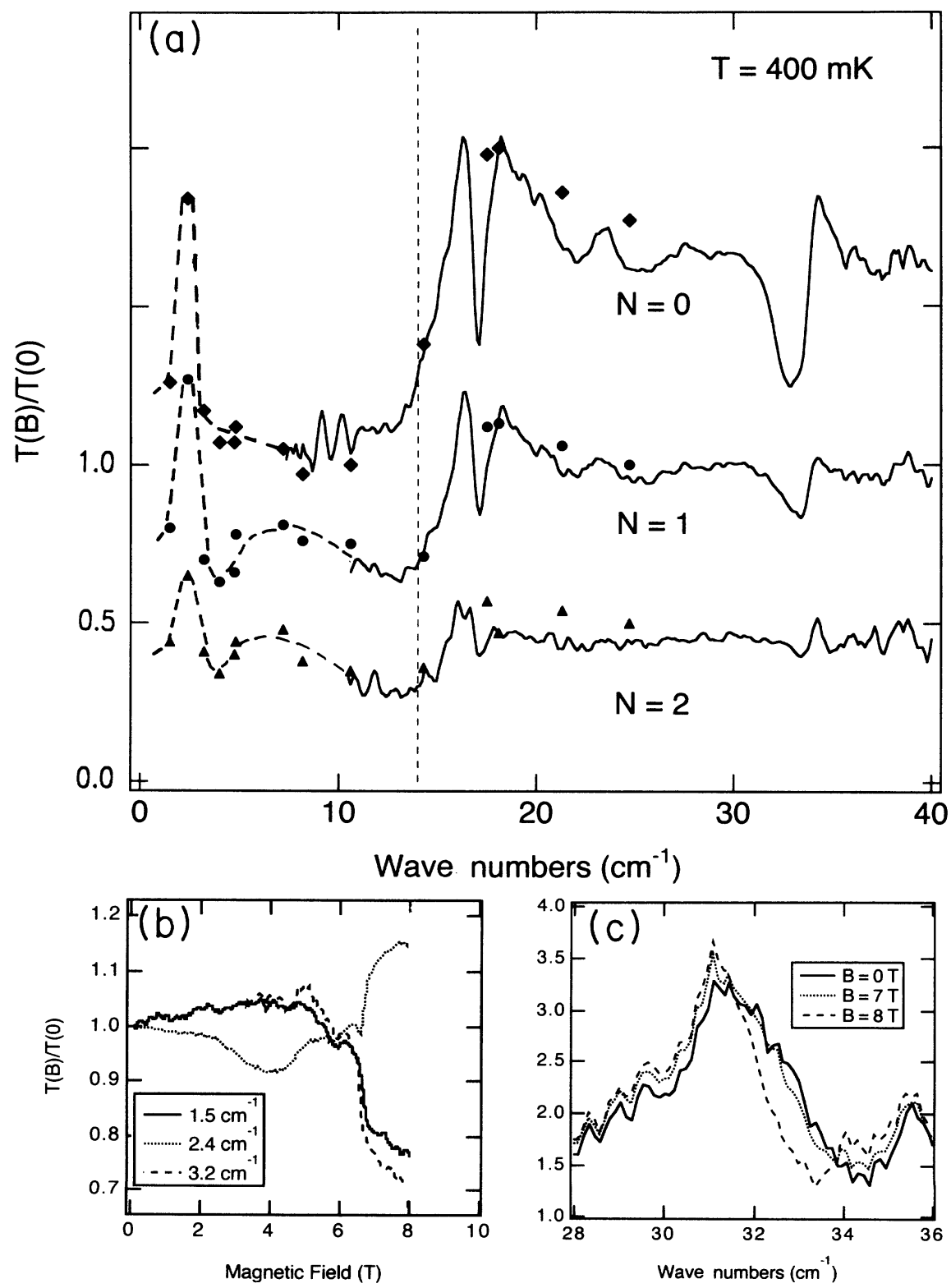

FIG. 2. (a) FIR spectra (normalized to the $B=0$ spectrum) for three different magnetic fields corresponding to the $N=0,1$, and 2 FISDW subphases. The microwave and FIR laser data are shown as points. The upper and lower spectrum have been offset by \pm 0.5 for clarity. (b) Field dependence of the normalized transmission through the grid in the microwave regime. (c) Enlarged part of the spectra around the $31-\mathrm{cm}^{-1}$ phonon (see text). 
The main feature in the spectra is the opening of a gap near $14 \mathrm{~cm}^{-1}$. This value is slightly higher than that estimated from the $1.2-\mathrm{K}$ reflectivity, ${ }^{13}$ indicating a moderate temperature dependence of the gap as expected in a mean-field treatment.

In a theoretical treatment to explain the quasiquantized Hall behavior, Montambaux, Heritier, and Leder$\mathrm{er}^{10}$ assumed a constant carrier density and concluded that a multiplet of gaps would form, separating Landaulike bands. As a consequence the magnitude of the gaps would be strongly field dependent and change discontinuously between the various SDW subphases.

The above results do not confirm this picture. We find no evidence for multiple gaps, and, more specifically, our data show that the fundamental gap is independent of the magnetic field or subphase index. However, the signal strength in the gap is not zero but does depend on the subphase index. We must conclude that the gap is not uniform in $k$ space.

To explain the data we propose the following simple qualitative model: When the field increases, the system will become more sensitive to a nesting instability, part of the charge carriers condense into a collective SDW ground state, and it will take a finite amount of energy (the gap) to create a free-electron excitation. In the original treatment of Gor'kov and Lebed"6 the free energy of the system is lowest if the area of the normal carrier pockets on the Fermi surface is quantized. The total gapped area, or equivalently the density of electrons in the collective state, will increase discontinuously upon each phase transition. (This agrees with the general observation of an increase of the magnetoresistance and a stepwise increase of the Hall voltage.)

The enhancement of the reflectivity above the gap is in accordance with a sum-rule conservation of the total oscillator strength for the electronic excitations, with only part of the total strength transferred to a collective mode. A somewhat surprising result is the fact that the collective mode appears at the rather high frequency of 2.4 $\mathrm{cm}^{-1}$. This could indicate a strong pinning of the electronic modulation to the lattice. Contrary to CDW systems, such a pinning is not expected for SDW.

The interaction of the SDW formation with the lattice is also obvious from the change in the phonon response, especially near $32 \mathrm{~cm}^{-1}$. This phonon is expected to be basically an acoustic mode, becoming FIR active because of the zone folding caused by the $\mathrm{ClO}_{4}$ anion ordering transition. ${ }^{15}$ This mode is depicted in more detail in Fig. 2(c), where we normalized the transmission signal to the reflection from the $a b$ plane, measured simultaneously. This mode is distorted from a simple Lorentzian, partly due to the varying dielectric constant in this frequency region. However, it is clear that, especially in the $N=0$ SDW state, the phonon line shape changes and becomes more asymmetric. This indicates an enhanced coupling of this phonon to the electronic system in the SDW state. Part of this coupling could result from the opening of the gap, which shifts electronic spectral weight to higher frequencies closer to the phonon frequency.

The origin of the feature around $17 \mathrm{~cm}^{-1}$ is not understood. One could speculate that the periodicity of the spin-density wave leads to additional minigaps in the phonon dispersion and induces new FIR-active modes that correspond to the original acoustic mode.

A final remark concerns the appearance of broad shallow dips in the field-dependent data shown in Fig. 1(a) (arrows). When compared with previous work at higher temperature, ${ }^{13}$ the number and sharpness of these features interpreted as a cyclotron resonance of free carriers is considerably reduced. We calculate the effective mass to be $m^{*}=0.7 m_{e}$, which is in the range of values found earlier.

In conclusion, we present spectroscopic evidence that the gap in the field-induced spin-density-wave state is independent of the value of the magnetic field. The data are consistent with a stepwise increase of the carrier density in the condensed SDW ground state upon each phase transition into a lower index subphase. In addition, we report the first indication of a collective mode excitation in the microwave region, and of an enhanced coupling of the $31-\mathrm{cm}^{-1}$ zone-folding phonon mode with the SDW formation.

This work was supported by the European Community, FOM, and NWO (the Netherlands), NSF Contract No. DMR 88-18510 and NATO Contract No. 0335/88. We are grateful to Paul Chaikin for providing the samples used.
"Present address: IBM Thomas J. Watson Research Center, P.O. Box 218 Yorktown Heights, NY 10598.

${ }^{1}$ See, e.g., reviews in Low Dimensional Conductors and Superconductors, edited by D. Jerome and L. Caro, Nato Advanced Study Institute, Series B: Physics, Vol. 155 (Plenum, New York, 1987).

${ }^{2}$ P. M. Chaikin et al., Phys. Rev. Lett. 51, 2333 (1983).

${ }^{3}$ K. Kajimura et al., J. Phys. (Paris) Colloq. 44, C3-1059 (1983).

${ }^{4}$ M. J. Naughton et al., Phys. Rev. Lett. 55, 969 (1985).

${ }^{5}$ F. Pesty, P. Garoche, and K. Bechgaard, Phys. Rev. Lett. 55, 2495 (1985).

${ }^{6}$ L. P. Gor'kov, and A. G. Lebed', J. Phys. (Paris) Lett. 45, L4333 (1984).
${ }^{7}$ M. Heritier, G. Montambaux, and P. Lederer, J. Phys. (Paris) Lett. 45, L943 (1984).

${ }^{8}$ G. Montambaux, M. Heritier, and P. Lederer, Phys. Rev. Lett. 55, 2078 (1985).

${ }^{9}$ K. Yamaji, J. Phys. Soc. Jpn. 54, 1034 (1985).

${ }^{10}$ G. Montambaux, and D. Poilblanc, Phys. Rev. B 37, 1913 (1988).

${ }^{11}$ D. Quinlivan et al., Phys. Rev. Lett. 65, 1816 (1990).

${ }^{12}$ G. Montambaux et al., Phys. Rev. B 39, 885 (1989).

${ }^{13}$ A. S. Perel et al., Phys. Rev. Lett. 67, 2072 (1991).

${ }^{14}$ H. K. Ng et al., J. Phys. (Paris) Lett. 43, L513 (1982).

${ }^{15}$ J. E. Eldridge et al., Phys. Rev. B 32, 5156 (1985). 